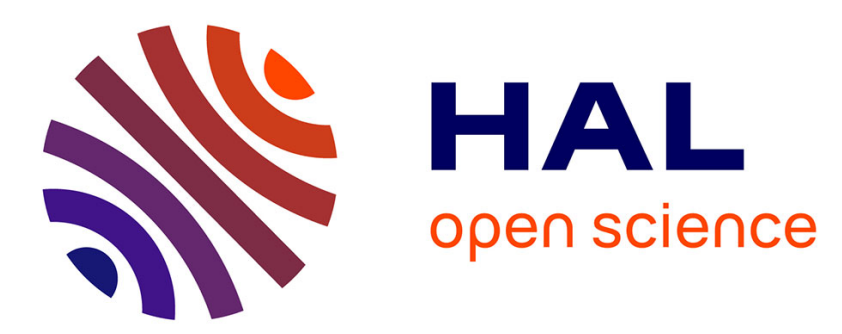

\title{
A Markov Point Process model for wrinkles in human faces
}

\author{
Nazre Batool, Rama Chellappa
}

\section{To cite this version:}

Nazre Batool, Rama Chellappa. A Markov Point Process model for wrinkles in human faces. 2012 19th IEEE International Conference on Image Processing (ICIP), Sep 2012, Orlando, United States. pp.1809 - 1812, 10.1109/ICIP.2012.6467233 . hal-01096679

\section{HAL Id: hal-01096679 https://hal.inria.fr/hal-01096679}

Submitted on 17 Dec 2014

HAL is a multi-disciplinary open access archive for the deposit and dissemination of scientific research documents, whether they are published or not. The documents may come from teaching and research institutions in France or abroad, or from public or private research centers.
L'archive ouverte pluridisciplinaire HAL, est destinée au dépôt et à la diffusion de documents scientifiques de niveau recherche, publiés ou non, émanant des établissements d'enseignement et de recherche français ou étrangers, des laboratoires publics ou privés. 


\title{
A MARKOV POINT PROCESS MODEL FOR WRINKLES IN HUMAN FACES
}

\author{
Nazre Batool, Rama Chellappa \\ Computer Vision Laboratory, Department of Electrical and Computer Engineering, \\ University of Maryland, College Park, MD, USA
}

\begin{abstract}
In this paper we present a new generative model for wrinkles on aging human faces based on Markov Point Processes (MPP) where wrinkles are considered as stochastic spatial arrangements of sequences of line segments. The model is then used in a Bayesian framework to localize the wrinkles in images. In aging human faces, wrinkles mostly appear as discontinuities in surrounding grayscale texture. The intensity gradients due to wrinkles are enhanced using filters and used as data to detect more probable locations and directions of line segments. Wrinkles are localized by sampling MPP using the Reversible Jump Markov Chain Monte Carlo (RJMCMC) algorithm. Experiments on images obtained from uncontrolled acquisition conditions are presented.
\end{abstract}

Index Terms - Wrinkles, Markov Point Process, Reversible Jump Markov Chain Monte Carlo, stochastic geometrical model

\section{INTRODUCTION}

Wrinkles and fine lines are important but subtle features of aging human faces. Localization/detection of wrinkles is a challenging problem due to physical properties of skin and image acquisition conditions. Wrinkles can be viewed roughly either as textures or edges depending on their appearances. Normally wrinkles create appearance of texture on skin. However, when looked at high resolution, wrinkles do not depict any specific repetitive/homogeneous texture pattern. Wrinkles cannot be described as boundaries between multiple textures as well. However, a wrinkle can be described as an artifact or a discontinuity in a surrounding inhomogeneous skin texture. Considering these specific characteristic appearances of wrinkles, we propose a novel generative modeling approach for wrinkles, based on intensity gradients and incorporating geometric properties of wrinkles as well.

Many applications in skin aging depend on the analysis of wrinkles as texture [1]. Some attempts have been made to evaluate wrinkles as edges for age detection. Kwon and Lobo [2] used active contour models to localize wrinkles in high resolution images. Recently, Cula et al. [3] reported work on localization and assessment of severity of facial wrinkles. The approach is based on enhancement of intensity gradients due to wrinkles using Gabor filters and subsequent thresholding of filter response. Our work follows the approach of the above mentioned methods by analyzing wrinkles as edges and is closest to the work by Soitca et al. [4]. We propose to incorporate semantic prior information available from typical appearance of wrinkles on human faces. The properties of wrinkles (angle, length, proximity) as prior information allow us to select edges that have higher probability of being wrinkles. The MPP model represents wrinkles as a stochastic spatial process of 'line segments' and uses prior knowledge to impose geometric constraints on line segments in a Bayesian framework.

\section{MARKOV POINT PROCESS}

A point process is defined with respect to Poisson measure (The interested reader is referred to [5] for details on MPP). A 'mark', which is a set of random parameters describing geometric properties, can be attached to a point. In this work, a point with a mark represents a line segment. Let a marked point (line segment) be denoted by $w_{i}=\left(s_{i}, m_{i}\right)$ where $s_{i}=\left(x_{i}, y_{i}\right) \in S \subset \mathbb{R}^{2}$ is the location of center of segment and $m_{i}=\left(l_{i}, \theta_{i}\right) \in M$ is the mark consisting of two parameters denoting length and orientation of the segment respectively. The continuous space for parameters is given by $M=\left[l_{\min }, l_{\max }\right] \times\left[\theta_{\min }, \theta_{\max }\right]$. The line segment sequences $\left\{\mathbf{w}=w_{i}, i=1, \ldots, n\right\}$ can be considered as realization of MPP on space $S \times M$. The probability density of MPP can be represented by the Gibbs distribution.

$$
\begin{aligned}
& f(\mathbf{w})=c \beta^{n(\mathbf{w})} \exp (-U(\mathbf{w})) \\
& f(\mathbf{w})=c \beta^{n(\mathbf{w})} \exp -\left(U_{P}(\mathbf{w})+U_{D}(\mathbf{w})\right)
\end{aligned}
$$

where $c$ is the normalizing constant, $\beta$ is the intensity of point process, $n(\mathbf{w})$ and $U(\mathbf{w})$ are the number of line segments and total energy for the realization $\mathrm{w}$ respectively. The total energy is the sum of two energy terms $U_{P}(\mathbf{w})$ and $U_{D}(\mathbf{w})$ corresponding to the prior model for interaction of line segments and data likelihood term respectively. 


\subsection{Prior Model}

The prior model captures more likely geometric properties of individual line segments (length and connectivity) as well as the interactions among neighboring line segments. The parameters $\left\{l_{i}, \theta_{i}\right\}$ associated with the mark $m_{i}$ are sampled from a Uniform distribution.

$$
l_{i} \sim \mathcal{U}\left(\left[l_{\min }, l_{\max }\right]\right) \text { and } \theta_{i} \sim \mathcal{U}\left(\left[\theta_{\min }, \theta_{\max }\right]\right)
$$

Let $q_{1}\left(w_{i}\right)$ and $q_{2}\left(w_{i}, w_{j}\right)$ denote the energies contributed by properties of an individual line segment $w_{i}$ and the interaction of two segments $\left\{w_{i}, w_{j}\right\}$. Then the total energy for the prior model is given by

$$
U_{P}(\mathbf{w})=-\sum_{w_{i} \in \mathbf{w}} \log q_{1}\left(w_{i}\right)-\sum_{\substack{\left(w_{i}, w_{j}\right) \in \mathbf{w} \\ w_{i} \sim w_{j}}} \log q_{2}\left(w_{i}, w_{j}\right)
$$

where $w_{i} \sim w_{j}$ denotes the interaction between two segments. The two individual geometric properties modeled are length and connectivity. Line segments with larger lengths are penalized as follows.

$$
q_{1, l}=\exp -\left(\frac{l-l_{\min }}{l_{\min }}\right)
$$

Regarding connectivity, a segment is allowed to be connected on either side by exactly one line segment which can result in singly or doubly connected segments. We want to penalize individual line segments with no connections at all. Let $c_{i} \in\{0,1,2\}$ denote the number of connections for the line segment $w_{i}$. The segments with more connections are favored as follows.

$$
q_{1, c}\left(w_{i}\right)=q\left(c_{i}+1\right)
$$

where:

$$
q=\left[\begin{array}{lll}
q(1) & q(2) & q(3)
\end{array}\right]^{T} \text { and } q(3) \geq q(2) \geq q(1)
$$

The interaction between line segments is modeled through the term $q_{2}\left(w_{i}, w_{j}\right)$. Two segments have rejection interaction if they are overlapping or lie within radius $r$ of each other. This penalizes the overlapping or congested line segments:

$$
q_{2}\left(w_{i}, w_{j}\right)=\gamma^{\mathbb{I}\left(w_{i} \sim w_{j}\right)}
$$

The parameter $\gamma$ is the penalty assigned to segments with rejection interaction and $\mathbb{I}_{\left(w_{i} \sim w_{j}\right)}$ is the indicator function for interacting segments. Figure 1 shows examples of interactions between two line segments.

\subsection{Data Likelihood}

The data likelihood captures the information on where line segments corresponding to wrinkles are more probable in the
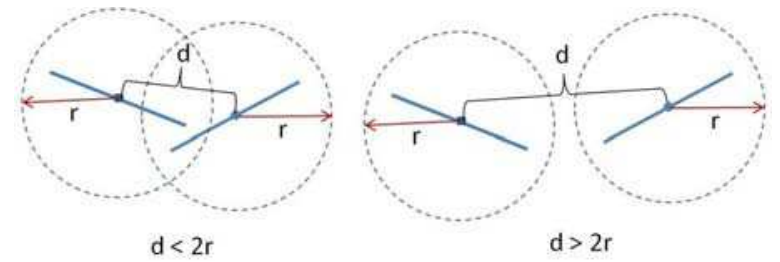

Fig. 1. (Left) Rejection Interaction (Right) No Rejection Interaction

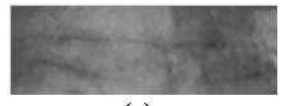

(a)

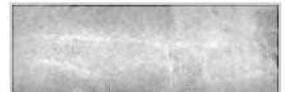

(c)

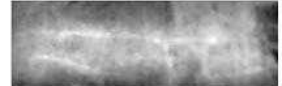

(b)

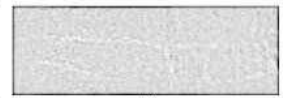

(d)
Fig. 2. LoG filter response to low resolution forehead image (a) Original Image (b) $\sigma=\frac{1}{2 \sqrt{2}}$ (c) $\sigma=\frac{1}{2}$ (d) $\sigma=\frac{1}{\sqrt{2}}$

given data. The images are filtered with Laplacian of Gaussian (LoG) filter to highlight intensity gradients caused by wrinkles. Let $D_{i}$ denote pixels in the image correspending to the segment $w_{i}$. The likelihood energy for the segment $w_{i}$ is given by

$$
U_{D}\left(w_{i}\right)=\alpha \sum_{d \in D_{i}}(d)
$$

Figure 2 shows results of LoG filter responses to low resolution forehead image for different values of variance. A trade off can be observed between localization of wrinkles and amplitude of LoG output to wrinkle gradients. We select the standard deviation $(\sigma=0.5)$ for LoG filters for this work.

\subsection{Simulation}

For MPP, the number of line segments is not known a priori and the Metropolis-Hastings algorithm cannot be used to sample from distributions. The algorithm is changed to allow configurations of different dimensions i.e. number of line segments. Green presented the Reversible Jump Markov Chain Monte Carlo algorithm to jump between states in configurations of different dimensions [6, 7]. Given that the state $\mathbf{w}$ is changed to $\mathrm{w}^{\prime}$, the algorithm requires matching of dimensions of two configurations. This is done using an auxilliary random variable $u$, sampled from a distribution $g(u)$, and a bijective transformation $\mathcal{T}$ such that $\mathbf{w}^{\prime}=\mathcal{T}(\mathbf{w}, u)$. The acceptance probability is then modified by the Jacobian of transformation as follows.

$$
\phi\left(\mathbf{w} \rightarrow \mathbf{w}^{\prime}\right)=\min \{1, R\}
$$

where $R$ is called acceptance ratio and given as

$$
R=\frac{f\left(\mathbf{w}^{\prime}\right)}{f(\mathbf{w}) g(u)}\left|\frac{\partial \mathcal{T}}{\partial(\mathbf{w}, u)}\right| \times \frac{\operatorname{prob}(\text { reverse jump })}{\operatorname{prob}(\text { forward jump })}
$$




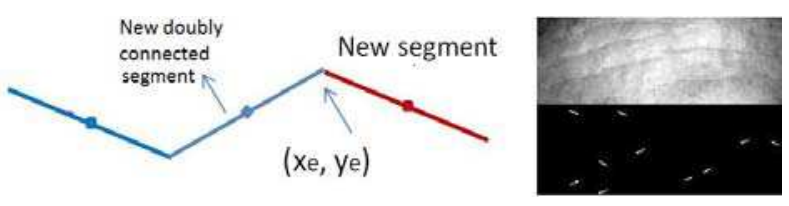

Fig. 3. (Left) Birth of a doubly connected segment (Right) Placement of Seed Segments

Simulated annealing is used to reach the global minimum of the Gibbs energy $U(\mathbf{w})$ in (2) where the probability density $f(\mathbf{w})$ is replaced by $f(\mathbf{w})^{\frac{1}{T_{i}}}$ in RJMCMC algorithm and $T_{i}$ denotes temperatue in iteration $i$. The simulation of point process involves the following four moves among configurations of different dimensions.

\section{Birth or Death of Free Segments}

\section{Birth or Death of Connected Segments}

Each move invovles the calculation of $g(u), \mathcal{T}(\mathbf{w}, u),\left|\frac{\partial \mathcal{T}}{\partial(\mathbf{w}, u)}\right|$ and $R$. For brevity, we skip the detailed derivation and present the final expressions for $R$ for different moves. Let $\left\{n, n_{c 0}\right.$, $\left.n_{c 1}, n_{c 2}\right\}$ denote the total number of segments and the number of segments having zero, one and two connections respectively. For the birth of a free segment, let $\omega$ denote the new free segment added to the configuration and let $u$ be the random vector sampled from the segment parameter space $(S, M)$ according to uniform distribution. Then $u$ is given by:

$$
u=\left[\begin{array}{llll}
x_{u} & y_{u} & l_{u} & \theta_{u}
\end{array}\right]^{T}
$$

and the density function for $u$ denoted by $g(u)$ is given as:

$$
g(u)=\frac{1}{\nu(S)} \times \frac{1}{l_{\max }-l_{\min }} \times \frac{1}{\theta_{\max }-\theta_{\min }}
$$

where $\nu(S)$ is the total measure on image space $S$. The bijective transformation for this move is selected to be:

$$
\mathbf{w}^{\prime}=\mathcal{T}(\mathbf{w}, u)=\left\{w_{1}^{\prime}=w_{1}, \ldots, w_{n}^{\prime}=w_{n}, \omega=u\right\}
$$

Then the Jacobian of the transformation is one. Let $P_{F, b i r t h}$ and $P_{F, \text { death }}$ be the probabilities of choosing birth and death of free segments respectively, $P_{F, \text { death }} / n_{c 0}$ is the probability of reverse jump of death of that particular free segment. Then, according to (11), the acceptance ratio for the birth of a free segment can be written as:

$$
R=\frac{P_{F, \text { death }}}{n_{c 0}} \times \frac{\nu(S)\left(l_{\max }-l_{\min }\right)\left(\theta_{\max }-\theta_{\min }\right)}{P_{F, \text { birth }}} \times \frac{f\left(\mathbf{w}^{\prime}\right)}{f(\mathbf{w})}
$$

where the ratio $\frac{f\left(\mathbf{w}^{\prime}\right)}{f(\mathbf{w})}=\frac{f(\mathbf{w} \cup \omega)}{f(\mathbf{w})}$ is given as follows:

$$
\frac{f\left(\mathbf{w}^{\prime}\right)}{f(\mathbf{w})}=\beta \exp \left(q_{1}(\omega) \sum_{\substack{w_{i} \in \mathbf{w} \\ w_{i} \sim \omega}} q_{2}\left(w_{i}, \omega\right)+\alpha \sum_{d \in D(\omega)} d\right)
$$

For the birth of a connected segment, a new segment is sampled and connected to a randomly selected segment, with at least one free end, from the configuration. This move can also result in doubly connected segments as is shown in Figure 3(a). For the death move, a singly connected segment is selected randomly which eventually can result in the deletion of a doubly connected segment. Let $\left(x_{e}, y_{e}\right)$ be the coordinates of the free end of an existing segment to connect the new segment to. Let $u=\left(\theta_{u}, l_{u}\right)$ be the random vector sampled from mark space $M$ with density function:

$$
g(u)=\frac{1}{l_{\max }-l_{\min }} \times \frac{1}{\theta_{\max }-\theta_{\min }}
$$

Then the new segment $\omega$ is given by:

$$
\begin{aligned}
\omega(u) & =\left[\begin{array}{llll}
x_{\omega}\left(x_{e}, \theta_{u}, l_{u}\right) & y_{\omega}\left(y_{e}, \theta_{u}, l_{u}\right) & \theta_{u} & l_{u}
\end{array}\right]^{T} \\
x_{\omega} & =x_{e} \pm \frac{l_{u}}{2} \cos \left(\theta_{u}\right) \text { and } y_{\omega}=x_{e} \pm \frac{l_{u}}{2} \sin \left(\theta_{u}\right)
\end{aligned}
$$

Then it can be shown that the Jacobian of the bijective tranformation is given as

$$
\left|\frac{\partial \mathcal{T}(\mathbf{w}, u)}{\partial(\mathbf{w}, u)}\right|=\left|\frac{\partial \mathbf{w}^{\prime}}{\partial(\mathbf{w}, u)}\right|=\left|\frac{\partial[\mathbf{w} \omega(u)]^{T}}{\partial(\mathbf{w}, u)}\right|=1
$$

Let $P_{C, \text { birth }}$ and $P_{C, \text { death }}$ be the probabilities of choosing the birth and death of a connected segment respectively, then $P_{C, b i r t h} /\left(2 n_{c 0}+n_{c 1}\right)$ is the probability of selection of a particular free end point $\left(x_{e}, y_{e}\right)$ and $P_{C, \text { death }} / n_{c 1}$ is the probability of reverse jump of death of a connected segment. Then the acceptance ratio for birth of connected segment can be written as:

$$
\begin{aligned}
R= & \frac{2 n_{c 0}+n_{c 1}}{P_{C, \text { birth }}} \times \frac{P_{C, \text { death }}}{n_{c 1}} \\
& \times\left(l_{\text {max }}-l_{\text {min }}\right)\left(\theta_{\text {max }}-\theta_{\text {min }}\right) \times \frac{f\left(\mathbf{w}^{\prime}\right)}{f(\mathbf{w})}
\end{aligned}
$$

where the ratio $\frac{f\left(\mathbf{w}^{\prime}\right)}{f(\mathbf{w})}$ is given in (16). The acceptance ratios for the reverse jumps i.e. death of a free or connected segments are simply the inverse of acceptance ratios of birth moves.

The connected segments connect to pre-existing segments with free end points making the placement of first few free segments critical in the evolution of Markov Chain in RJMCMC algorithm. For faster convergence towards the global minimum, we use a favorable initial state by introducing 'seed' segments. These segments are free segments placed in the image at high LoG filter responses. The image sites with highest filter responses are selected while keeping a minimum distance among them. Seed segments are then placed on these sites in the direction perpendicular to the highest intensity gradient. Figure $3($ b) shows an example of placement of seed segments. 

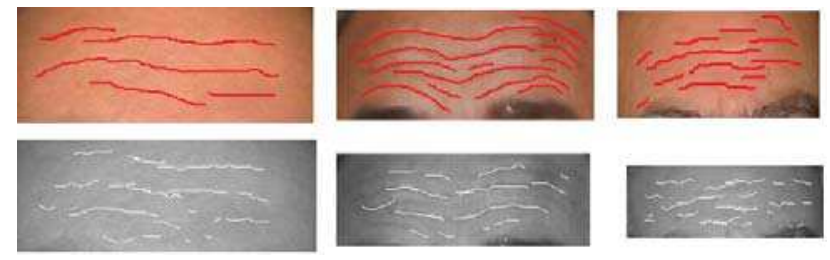

Fig. 4. (Top) Ground Truth (Bottom) localization results

\begin{tabular}{|l|l|l|l|l|l|}
\hline$\alpha$ & $\beta$ & $\gamma$ & $\log q_{c 0}$ & $\log q_{c 1}$ & $\log q_{c 2}$ \\
\hline \hline 4000 & 0.005 & 5000 & $-10^{10}$ & $10^{6}$ & $10^{10}$ \\
\hline
\end{tabular}

Table 1. Parameter Values

\section{RESULTS}

Experiments were conducted on face images from the FG-Net [8] dataset having various resolution and illunination settings. Each move in RJMCMC algorithm was selected with equal probabiliy i.e. 1/4 where one iteration performed one move only. Table 1 shows selected values for different parameters of the MPP model. Figure 5 shows localization results for different subjects. It can be seen that, despite low resolution, most of the wrinkles are detected with few false alarms. Since the ground truth is not available, some of the images were used to draw wrinkles by hand. Figure 4 shows the comparison of groud truth vs. localization results for those images. It can be observed that the localized wrinkles closely resemble the ground truth. However, the line sequences are broken at some places and do not cover the original wrinkle completely. At some locations, some overlapping of segments can also be observed.

\section{CONCLUSION}

The main contribution of this work is the modeling of wrinkles as spatial line process to incorporate specific prior knowledge about wrinkles. The experiments on images with uncontrolled acquisition conditions highlight the strength of our modeling approach. This work can be the basis for applications based on modeling of wrinkles. In future, we plan to extend the current work by using more sophisticated geometric model and estimation of model parameters.

\section{ACKNOWLEDGMENT}

The first author would like to acknowledge the support of Fulbright/HEC(Pakistan)/USAID PhD Scholarship. However, neither the governments of the United States/Pakistan nor any agencies representing them have endorsed the conclusions or approved the contents of this publication.
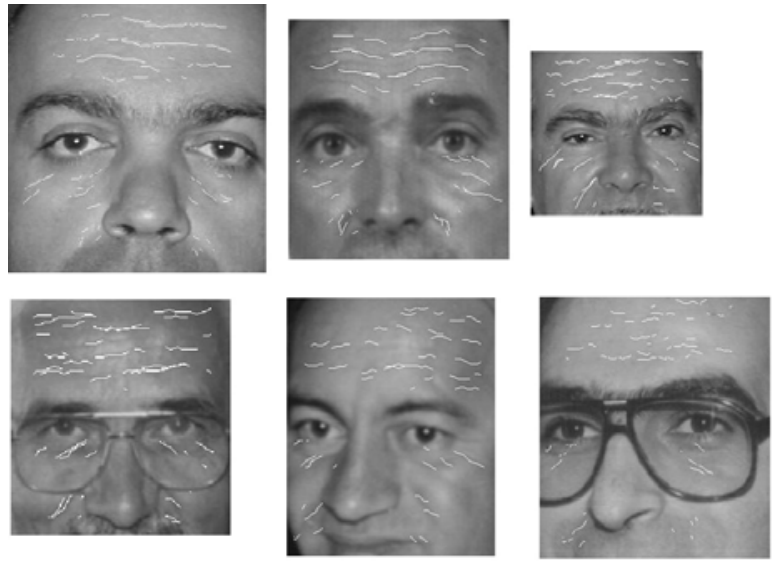

Fig. 5. Localization results

\section{REFERENCES}

[1] Yun Fu, Guodong Guo, and T.S. Huang, "Age synthesis and estimation via faces: A survey," Pattern Analysis and Machine Intelligence, IEEE Transactions on, vol. 32, no. 11, pp. 1955-1976, nov. 2010.

[2] Young H. Kwon and Niels Da Vitoria Lobo, "Age classification from facial images," in Proc. IEEE Conf. Computer Vision and Pattern Recognition, 1999, pp. 762-767.

[3] Gabriela O Cula, Paulo R Bargo, and Nikiforos Kollias, "Assessing facial wrinkles: automatic detection and quantification," Proceedings of SPIE, vol. 7161, pp. 71610J-71610J-6, 2009.

[4] Radu Stoica, Xavier Descombes, and Josiane Zerubia, "A Gibbs point process for road extraction from remotely sensed images," Int. J. Comput. Vision, vol. 57, pp. 121136, May 2004.

[5] M. N. M. Lieshout, Markov Point Processes and their Applications, Imperial College Press/World Scientific Publishing: London/Singapore, 2000.

[6] P. Green, "Reversible jump MCMC computation and Bayesian model determination," Biometrika, vol. 82, pp. 711-732, 1995.

[7] C. J. Geyer and J. Mller, "Simulation procedures and likelihood inference for spatial point processes," Scandinavian Journal of Statistics, vol. 21, no. 4, pp. 359-373, 1994.

[8] "The FG-net aging database," http://www.fgnet.rsunit.com/. 